\title{
On Majoritarian Bargaining with Incomplete Information
}

\author{
Tsung-Sheng Tsai* \\ Department of Economics, National Tsing Hua University \\ C.C. Yang \\ Institute of Economics, Academia Sinica \\ Department of Public Finance, National Chengchi University \\ Institute of Economics, National Sun Yat-sen University
}

December 2008

[Abstract] This paper studies a finite horizon version of Baron and Ferejohn's (1989) majoritarian bargaining with incomplete information. Our devised model essentially blends Spence's signaling and the coalition formation of majoritarian bargaining. The main findings include: (i) oversized coalitions may arise in equilibrium and allowing for delay can be optimal for a proposer; (ii) whether being regarded as a high type makes a player better or worse off is not predetermined a priori but depends on two conflicting concerns: the “offer” concern à la Spence’s signaling (the higher the type, the higher the offer) and the "coalition” concern in majoritarian bargaining (the higher the type, the lower the probability of being a member of the majoritarian coalition); (iii) players take action to distinguish themselves via delay; however, separating equilibria often fail to exist due to the incapability or unprofitability of exercising separation; (iv) separating equilibria, if they exist, can have a "no-envy" property, and pooling equilibria may coexist with the "least-cost" separating equilibrium even after refining the equilibrium set by the intuitive criterion; and (v) equilibria may be non-stationary and exhibit the novel feature that pooling proposals arise if a game lasts an even number of sessions, but not if there is an odd number of sessions.

JEL classification: C78, D72, D82.

\footnotetext{
* Corresponding author, Tsung-Sheng Tsai. Mailing address: Department of Economics, National Tsing Hua University, Hsinchu 300, Taiwan. E-mail: tstsai@mx.nthu.edu.tw.
} 


\section{Introduction}

In an important paper, Baron and Ferejohn (1989) introduced a majoritarian bargaining model (henceforth referred to as the BF model) that extended the Stahl (1972)-Rubinstein (1982) model to a setting where not all players have to agree in order to implement a proposal. This model has been applied widely, and is considered to be a workhorse in the study of various political issues. ${ }^{1}$

Like the original Stahl-Rubinstein model, the BF model is a game with complete information. While many studies have analyzed incomplete information extensions of the Stahl-Rubinstein model, ${ }^{2}$ little is known about majoritarian bargaining under incomplete information. This paper seeks to take a step to fill this void.

Players are assumed to have a common discount factor in the BF model. BF offered two interpretations for the discount factor: (i) the political imperative derived from players' reelection concerns to distribute benefits sooner rather than later, and (ii) the probability that a player will be reelected in the next election. Following either interpretation, it seems natural to allow for heterogeneous discount factors among players. This paper considers such heterogeneity in the simplest manner possible: a player's discount factor is either high (a high type) or low (a low type). Our key assumption is that a player's discount factor is private information and unknown to other players. This incomplete information assumption can be justified on the grounds that a legislator knows the preferences of her own constituency better than other legislators.

When the number of bargaining sessions is finite and a motion is voted on immediately (i.e., the closed rule), the BF model yields two main predictions: ${ }^{3}$

1. Minimal size of coalitions - only a minimal majority of players receive a positive allocation of benefits from the bargaining.

\footnotetext{
${ }^{1}$ See Persson and Tabellini (2000) for applications and references.

${ }^{2}$ For a recent survey of this literature, see Ausubel et al. (2002).

${ }^{3}$ These two predictions will be preserved in an infinite-session setting if equilibria are confined to being stationary, but they will not hold in general if equilibria are non-stationary (see BF for the detail). The closed rule is a restrictive procedure, which does not permit amendments on the floor. Sinclair (1995) documented that some types of restrictive rules have been employed with increasing frequency in the U.S. Congress, reaching 66\% of the bills in the $102^{\text {nd }}$ Congress.
} 
2. No delay - the motion receives a majority support and bargaining ends in the first session.

Putting these two properties together gives rise to the so-called "minimum winning coalition" (MWC) - with "minimum” being for the first property and "winning” for the second property. As we will demonstrate, both these properties may fail in the incomplete information version considered in our paper.

Besides a two-type model, most of our paper considers a version with three players and three sessions. While this is a limitation, it turns out that the setup is rich enough to capture several interesting aspects of how incomplete information affects majoritarian bargaining. Obviously, the type space is the smallest possible, but the model is also "minimal" in the sense that: (i) we need at least three players for any non-trivial majority rule bargaining to take place, and (ii) any information revealed in the first session is relevant in the next session only if there is at least one additional round of bargaining. Fully understanding this minimal model should be a necessary step toward understanding more general versions of majority rule bargaining with incomplete information. Importantly, as will be seen, our devised model essentially blends Spence’s signaling and the coalition formation of majoritarian bargaining.

We show that the characterization of equilibrium proposals crucially depends on the difference between the discount factors of the two types. When the difference is small, all players form minimum winning coalitions. However, when the difference is large enough, oversized coalitions arise. In this case, delay occurs with positive probability in equilibrium. The logic for this result is based on a simple tradeoff. Making sure that a proposal will pass with certainty may be costly, since one responder must be given an offer that is large for both types to accept. Given that the difference between types is large enough, it will therefore be better to gamble and buy two risky votes by offering the two responders the continuation value of the impatient type. $^{4}$ The downside is that the proposal is rejected if both responders are of the patient type. However, from the ex ante point of view, this will nevertheless be

\footnotetext{
${ }^{4}$ We show in our model that it is never optimal to gamble by buying only one risky vote.
} 
optimal for a proposer as long as the impatient players are sufficiently cheap to buy. This tradeoff can be viewed as a simple formulation of Riker (1982), where it is argued that oversized coalitions are formed in order to reduce or avoid the uncertainty of losing the political battle. In our model this uncertainty is explained endogenously because of the tradeoff between the probability of winning and the share of benefits that goes to the proposer.

In Spence-style signaling models, both high and low types want to be regarded as a high type, as the wage payment is monotonically increasing in the expected type. In contrast, as pointed out by Harrington (1990), Persson and Tabellini (2000, p. 39) and others, being regarded as a low type may be beneficial when bargaining requires only a majority consent. This is because higher types have higher continuation values, implying that their reservation prices are higher. However, this may be bad for the high type players, since it may decrease the probability of being included in a winning coalition in majoritarian bargaining.

Both effects matter in our model. Conditional upon being included in a coalition, it is better to be regarded as a high type as this means that the share of the benefits will be higher. However, being regarded as a low type increases the probability of coalition inclusion. Which of these two effects is the more important depends on the parameters of the model.

Being regarded as some type is better or worse off for a player is one thing. Whether it is capable and profitable for the player to signal her type is another thing. In our model, acceptance ends the game and hence the only way that private information can be revealed in equilibrium is via players' exercising delay. Since patient players are more tolerant toward delay than impatient players, it is impossible for a low type to take action to distinguish herself from a high type: every delay action taken by the low type can be imitated by the high type in a cheaper way.

Even if it is better to be revealed as a high type than a low type, delay could be too costly and hence it may not be profitable for the high type to delay acceptance in order to separate. We show that separating equilibria exist only if the difference 
between the types is neither too big nor too small. If a separating equilibrium exists, a high type proposes an oversized coalition, which will lead to delay with positive probability, whereas a low type proposes a minimum winning coalition, which is accepted for sure. Interestingly, separating equilibria can have a "no-envy” property, in which case each type is able to choose a proposal under which none of the incentive compatibility constraints are binding. In addition, standard refinements do not have the same bite in our setup. We show that pooling equilibria may coexist with the "least-cost" separating equilibrium even after refining the equilibrium set using the intuitive criterion.

We also extend the model beyond three sessions, in which two new features emerge. First, which player type has an advantage in a given session may alternate over time. This is because strong players in the next session are more expensive to buy, so they have higher probabilities of being excluded from the coalition formation in the current session, which makes them weak. Second, Norman (2002) found that this logic implies that proposals typically do not converge in a perturbed version of the BF model with complete information. We also obtain equilibria that are non-stationary for similar reasons. However, our equilibria have the novel feature that pooling proposals arise if a game lasts an even number of sessions, but not if there is an odd number of sessions.

The remainder of the paper is organized as follows. Section 2 introduces our model. Section 3 analyzes the "minimal" model in detail and Section 4 extends the model beyond three sessions. Section 5 concludes.

\section{The model}

Our model is the stylized closed-rule version of the BF model with the peculiarity that the information assumption is relaxed from complete to incomplete.

There are 3 players (say, legislators) who must decide how to divide a dollar among themselves. This “divide-the-dollar” game lasts a finite number of sessions. Within each session, a player has a probability $1 / 3$ of being recognized, and if 
recognized, makes a proposal that divides the dollar. This recognition rule is common knowledge among the players. The proposal made is publicly known and voted on immediately. If it receives a majority support, the dollar is divided accordingly; if it fails to receive a majority support, the game moves on to the next session. All players will earn a zero payoff if no proposal receives a majority support before the end of the last session.

Let $x^{i}(t)=\left(x_{1}^{i}(t), x_{2}^{i}(t), x_{3}^{i}(t)\right)$ with $\quad x_{j}^{i}(t) \geq 0 \quad$ and $\quad \sum_{j=1}^{3} x_{j}^{i}(t)=1$ denote a proposal made by player $i$ in session $t$. The preferences of player $j$ over $x^{i}(t)$ are represented by the utility function $u^{j}\left(x^{i}(t)\right)=\rho^{t-1} x_{j}^{i}(t)$, where $0<\rho \leq 1$ denotes the discount factor. A player's discount factor $\rho$ is private information and equal to either $\bar{\rho}$ or $\underline{\rho}$ with $\bar{\rho}>\underline{\rho}$. A player who possesses $\bar{\rho}$ is called the patient or high $(\mathrm{H})$ type, while a player who possesses $\underline{\rho}$ is called the impatient or low (L) type. Either type is equally likely a priori. The utility function and the prior probability regarding types are common knowledge.

Clearly, assuming that there are only three players, that players are equally likely to propose, and that the two types are equally likely are all restrictions. However, as will be seen, the scenario is sufficiently rich and complex to yield novel and interesting results.

A strategy for a player lists in each session a prescription as to what proposal to make if she is recognized and of how to vote when a vote is required. Players are not able to make binding commitments when proposing or voting. The players' voting record in a session is publicly known after the session. Let $v_{j}(t)$ denote the ex ante value of the game to player $j$ at the beginning of session $t$ if the game continues to session $t$. Thus $\rho v_{j}(t)$ is player $j$ 's continuation value if viewed from session $t-1$. In general, the value of $v_{j}(t)$ will be history dependent, where a 
"history" at the beginning of session $t$ is given by the players' action profiles in previous sessions, including the identity of the proposers, the proposals made, and the voting records. We will restrict attention to equilibria in pure strategies. ${ }^{5}$

An equilibrium of the game is a profile of players' strategies that satisfies: ${ }^{6}$

(a) Sequential rationality: given players' beliefs about other players' types, (i) player $i$, if she becomes a proposer in session $t$, will choose a proposal $x^{i}(t)$ to maximize her expected utility, and (ii) player $j$ will vote for the proposal $x^{i}(t)$ if and only if $x_{j}^{i}(t) \geq \rho v_{j}(t+1) ;^{7}$

(b) Bayes' consistency of beliefs: players' beliefs are consistent with the equilibrium strategies and are determined by Bayes’ rule where possible.

In signaling-style games, there typically exists a multiplicity of equilibria due to the arbitrariness of beliefs off the equilibrium path. We shall apply commonly used reasonable-beliefs refinements to restrict players' off-the-equilibrium-path beliefs whenever appropriate.

For simplicity, we shall only report the case where $\bar{\rho}=1$ and $\underline{\rho}=\delta$. This case is representative of the more general setting with $0<\underline{\rho}<\bar{\rho} \leq 1$.

We need to make one remark before proceeding with the analysis. If a proposer wants to seek a coalition partner only, she may be indifferent over which of her two responders to include in the coalition. There are many ways of breaking the tie. For example, she may break the tie symmetrically, so that the two responders are equally likely to be chosen, or she may break the tie asymmetrically, say, by choosing one of the two responders with certainty. Norman (2002) first pointed out that the way in which ties are broken could exert a significant effect on the equilibrium outcomes. Indeed, in the finite-session version of the BF model he proved a folk-style theorem: a continuum of divisions of the dollar is supportable as subgame

\footnotetext{
${ }^{5}$ However, we exogenously impose a symmetric way of breaking ties; see below.

${ }^{6}$ More precisely, it is known as a perfect Bayesian equilibrium; see Mas-Colell et al. (1995).

${ }^{7}$ A player may violate (ii) if she is not pivotal in voting. As in BF, we consider a refinement in which weakly dominated strategies are eliminated.
} 
perfect equilibria as long as there are three or more sessions. To focus on the impact of incomplete information, we shall deliberately abstract from this complication and assume that players always break ties symmetrically when indifference is involved and that this practice of breaking ties is common knowledge among the players. For ease of exposition, however, we will only report the case where a responder indexed with a lower subscript is chosen as the coalition partner ex post whenever a proposer is indifferent over which of her two responders to include in the coalition. For example, suppose that player 1 is the proposer and that she is indifferent over players 2 and 3 as her coalition partner. Then we always report the case where player 2 is chosen ex post.

\section{Three-session game}

This section analyzes the "minimal” model, that is, the case where there are three players and the game lasts three sessions. The focus will be on the characterization of the equilibrium proposal in the first session. We solve the game backwards. As a benchmark, we simply note that the MWC prediction would hold in the "minimal" model if information were complete. ${ }^{8}$

\subsection{Third session}

The game ends with a zero payoff for everybody if no proposal receives a majority support at the end of the third (last) session. With sequential rationality, players will vote for any proposal in the third session. This implies that the proposer who is recognized in the third session will allocate the whole dollar to herself, regardless of the information revealed in previous sessions and her type being $\mathrm{H}$ or $\mathrm{L}$. Thus the ex ante value of the game to any player at the beginning of the third session equals $1 / 3$.

\footnotetext{
${ }^{8}$ However, the MWC prediction with complete information depends on symmetric tie-breaking; see Norman (2002).
} 


\subsection{Second session}

Since players' posteriors at the beginning of the third session do not have any impact on their behavior in the third session, it is clear that there is no room for players to signal their types in the second session of the game. No role for signaling implies that there will be no mimicking between the types. Thus, regardless of whether she is a type $\mathrm{H}$ or $\mathrm{L}$, and which history she is at, a proposer in the second session only needs to consider the following three proposals: (i) allocate a type H's continuation value $(1 / 3)$ to one responder and zero to the other, (ii) allocate a type L's continuation value $(1 / 3) \delta$ to one responder and zero to the other, and (iii) allocate a type L's continuation value $(1 / 3) \delta$ to both responders. We shall call the three proposals above $1 h, 1 \ell$ and $2 \ell$, respectively. Proposals other than these three are strictly dominated by one of these proposals: they give the proposer a lower share of the dollar, but do not increase the probability of winning a majority support.

Let $p=\left(p_{1}, p_{2}, p_{3}\right)$ with $p_{j}$ denote the common posterior at the beginning of the second session that players other than $j$ attach to the possibility that player $j$ is a type H. Thus, player 1's belief is represented by $\left(p_{2}, p_{3}\right)$, player 2 's by $\left(p_{1}, p_{3}\right)$, and player 3's by $\left(p_{1}, p_{2}\right)$. The posterior is common because players other than $j$ share the same information regarding $j$ and hence they have the same belief regarding $j$. By definition, we allow for the possibility that $p_{j}=1$ but player $j$ herself is a type L.

The following lemma prescribes a player's optimal proposals in the second session if she becomes the proposer. Since we restrict attention to characterizing separating and pooling proposals (but not semi-pooling) in the first session, this makes the posterior about player 1 (the proposer in the first session, see below) only take on three values: 0,1 and 1/2. Lemma 1 only reports those $p$ 's which will be utilized in the derivation of separating and pooling proposals. 
Lemma 1. Players' proposal choices in the second session are as follows:

1. If $p=(1,1,1),(1,1,1 / 2)$ or $(1 / 2,1,1)$, all players will choose $1 \mathrm{~h}$.

2. If $p=(0,1,1)$ or $(0,1,1 / 2)$, player 1 will choose $1 \mathrm{~h}$ while both players 2 and 3 will choose $1 \ell$ with player 1 as their coalition partner.

3. If $p=(1 / 2,1,1 / 2)$, player $j$ with $p_{j}=1 / 2$ will choose $1 \mathrm{~h}$ while player $j$ with $p_{j}=1$ if she is a type $H$ will choose 1 h over $2 \ell$ if and only if $\delta \geq 1 / 3$, but if she is a type $L$ will choose 1 h over $2 \ell$ if and only if $\delta \geq 1 / 5$.

Proof:

Without loss of generality, assume that player 1 becomes the proposer in the second session. Given the posterior $p$, without loss let $p_{2} \leq p_{3}$. If player 1 is a type $H(L)$, her expected payoffs from choosing $1 h, 1 \ell$ and $2 \ell$ equal, respectively $1-(1 / 3), \quad(1-(1 / 3) \delta)\left(1-p_{2}\right)+(1 / 3) p_{2}$, and $(1-(2 / 3) \delta)\left(1-p_{2} p_{3}\right)+(1 / 3) p_{2} p_{3} ;$ $1-(1 / 3), \quad(1-(1 / 3) \delta)\left(1-p_{2}\right)+(1 / 3) \phi_{2}$, and $(1-(2 / 3) \delta)\left(1-p_{2} p_{3}\right)+(1 / 3) \dot{p}_{2} p_{3}$.

With sequential rationality, player 1 will choose the proposal that maximizes her expected payoff. The results stated in Lemma 1 are obtained when the relevant $p$ 's are substituted into the above payoffs. Q.E.D.

Note that $p_{j}(j \neq 1)$ only takes on two values 1 and $1 / 2$ in Lemma 1 . We explain the reason below.

\subsection{First session}

Without loss let player 1 be the proposer in the first session. There are three different sets of candidates for $x^{1}(1)$ : (i) seek a type H's support (i.e., buy one sure yea vote since the targeted responder is supposed to accept the offer regardless of whether she is a type H or L), (ii) seek a type L's support (i.e., buy one risky yea vote since the targeted responder may turn out to be a type $\mathrm{H}$ and so reject the offer), and (iii) seek the support of two type Ls (i.e., buy two risky yea votes since both 
responders may turn out to be a type $\mathrm{H}$ and so reject the offer). We shall call these three sets of proposals, respectively, $1 h(\mathrm{H}), 1 \ell(\mathrm{H})$ and $2 \ell(\mathrm{H})$ if player 1 is a type $\mathrm{H}$, and $1 h(\mathrm{~L}), 1 \ell(\mathrm{L})$ and $2 \ell(\mathrm{L})$ if she is a type $\mathrm{L}$. In the case where $1 h(\mathrm{H})=1 h(\mathrm{~L})$, $1 \ell(\mathrm{H})=1 \ell(\mathrm{L})$ and $2 \ell(\mathrm{H})=2 \ell(\mathrm{L})$, we shall call these pooling proposals $1 h(\mathrm{HL})$, $1 \ell(\mathrm{HL})$ and $2 \ell$ (HL), respectively.

For the "risky" proposals $1 \ell($.$) and 2 \ell($.$) , the game will continue to the second$ session with a positive probability in equilibrium. Under sequential rationality, their rejections if they occur imply that the targeted responders who reject the proposals must be a type H. As to the "sure" proposals $1 h($.$) , the second session is not$ supposed to occur in equilibrium since they seek one sure yea vote. However, if the second session occurs unexpectedly, we assume throughout that off-the-equilibrium-path beliefs regarding the identity of players' types are "monotonic," in the sense that a better offer that receives a rejection should not persuade players to believe that the responder is more likely to be a type $L$ than a type H. For example, if $p_{2}=1$ after the proposal $(1-(1 / 3) \delta,(1 / 3) \delta, 0)$ is rejected by

player 2, then players must believe that $p_{2}=1$ as well if the proposal $(1-(1 / 3),(1 / 3), 0)$ is rejected by player 2 . The monotonicity assumption first appears in Rubinstein (1985).

\subsubsection{No signaling}

It is useful to first consider what would happen if player 1's type were publicly known so that there were no signaling consideration for the proposer.

No signaling consideration implies that there would be no need to take into account the incentive compatibility (IC) constraints of mimicking between the types. As a result, similar to the second session, a proposer only needs to consider the following three proposals: (i) allocate a type H's continuation value to one responder and zero to the other, (ii) allocate a type L's continuation value to one responder and zero to the other, and (iii) allocate a type L's continuation value to both responders. We call these specific proposals the best of $1 h(\mathrm{H}), 1 \ell(\mathrm{H})$ and $2 \ell(\mathrm{H})$, respectively, if 
player 1 is a type $\mathrm{H}$, and the best of $1 h(\mathrm{~L}), 1 \ell(\mathrm{L})$ and $2 \ell(\mathrm{L})$, respectively, if player 1 is a type L. These proposals are "best" in the sense that they allocate merely continuation values to the targeted responders and zero to the rest. Put differently, these proposals merely satisfy the "individual rationality” constraints in a sense.

The following two propositions summarize our "no signaling" finding. ${ }^{9}$

Proposition 1. Suppose that the type of the proposer (player 1) is known to be high. Then, in any equilibrium with monotone beliefs:

a. The best of $1 h(\mathrm{H}),(2 / 3,1 / 3,0)$, is proposed in Session one if $\delta>1 / 3$.

b. The best of $2 \ell(\mathrm{H}),(1-(2 / 3) \delta,(1 / 3) \delta,(1 / 3) \delta)$, is proposed in Session one if $\delta<1 / 3$.

When compared with the MWC prediction of the BF model, the most interesting finding of Proposition 1 is the adoption of the $2 \ell(\mathrm{H})$ proposal: oversized coalitions arise and, moreover, delay occurs with positive probability. We show later that this finding remains true with signaling.

What is the motivation for players to form oversized coalitions? Riker (1962, p. 48) provided a celebrated answer:

"Since the members of a winning coalition may be uncertain about whether or not it is winning, they may in their uncertainty create a coalition larger than the actually minimum winning coalition.”

Consider the best of $2 \ell(\mathrm{H})$ (an over-sized coalition) versus the best of $1 h(\mathrm{H})$ (a minimal-sized coalition). When the value of $\delta$ is small $(\delta<1 / 3)$, it is more profitable in the ex ante sense for a type $\mathrm{H}$ to offer the (relatively small) (2/3) $\delta$ share of the dollar to buy two risky votes than to offer the (relatively large) $1 / 3$ share of the dollar to buy one sure vote. The cost paid for this ex ante "cheaper"

\footnotetext{
${ }^{9}$ For simplicity, we do not specify the borderline case. All proofs are relegated to the Appendix. A more detailed version of the proofs can be found on the website: http://mx.nthu.edu.tw/ tstsai/
} 
oversized coalition is that delay occurs with positive probability in equilibrium: there is a 1/4 chance that both responders will turn out to be of the $\mathrm{H}$ type ex post, so the offer is rejected. In contrast, rejection or delay would not arise if the more expensive proposal, the best of $1 h(\mathrm{H})$, were to be offered instead. It is arguable that the tradeoff between the share of the "pie" offered to coalition members and the probability of winning may be the key idea underlying Riker's answer to the formation of oversized coalitions.

Proposition 2. Suppose that the type of the proposer (player 1) is known to be low. Then, in any equilibrium with monotone beliefs:

a. The best of $1 h(\mathrm{~L}),(11 / 18+(1 / 9) \delta, 7 / 18-(1 / 9) \delta$, 0$)$, is proposed in Session one if $\delta>\delta_{0}$

b. The best of $2 \ell(\mathrm{L}),\left(1-(7 / 9) \delta+(2 / 9) \delta^{2},(7 / 18) \delta-(1 / 9) \delta^{2},(7 / 18) \delta-(1 / 9) \delta^{2}\right)$, is proposed in Session one if $\delta<\delta_{0}$,

where $\delta_{0}=(23-3 \sqrt{41}) / 16 \approx 0.237$.

The logic underlying Proposition 2 is similar to that in the case of Proposition 1, except that continuation payoffs are different since the L-type proposer will be more likely to be included in a winning coalition following a rejection (see Lemma 1).

Propositions 1 and 2 show that the equilibrium proposals vary from $1 h($.) to $2 \ell$ (.) as the value of $\delta$ decreases. This result is intuitive. When $\delta$ is relatively large ( $\delta>1 / 3$ ), the price of buying one sure vote (i.e., paying a type H's continuation value) does not differ much from the price of buying one risky vote (i.e., paying a type L's continuation value). The probability of failing to receive a majority support is the dominating concern for a proposer in such a situation. This explains why the best of $1 h($.) is the proposal for both types if $\delta>1 / 3$. By contrast, when the value of $\delta$ is relatively small ( $\delta<\delta_{0} \approx 0.237$ ), the price of buying one sure vote will be 
much higher than the price of buying one risky vote. The price of buying votes now becomes the dominating concern for a proposer. It turns out that it is optimal ex ante for both types to buy two risky votes rather than one sure vote. This results in the choice of the best of $2 \ell$ (.) for both types if $\delta<\delta_{0}$. In-between $\delta_{0} \leq \delta \leq 1 / 3$, the separation toward delay occurs, so a type $H$ prefers $2 \ell(H)$, while a type $L$ prefers $1 h(\mathrm{~L})$. This is because a type $\mathrm{H}$ is more tolerant toward delay than a type $\mathrm{L}$ : delay is costless for a type $\mathrm{H}$, but costly for a type L.

\subsubsection{Signaling}

We now consider the more realistic situation where player 1's type is private information, unknown to other players. In this world, it is possible for player 1 to signal her type via proposing behavior and hence we need to consider the IC constraints of mimicking between the types. Taking into consideration the IC constraints implies the possible existence of "information rent." In other words, buying one sure vote may require the allocation of a share of the dollar that is higher than a type H's continuation value. Similarly, buying one risky vote may require the allocation of a share of the dollar that is higher than a type L's continuation value.

What is the signaling mechanism in our model? Put differently, how may a player take action to distinguish herself from the other type of players if she wishes to do so? A crucial setting of our model is that $\bar{\rho}=1$ and $0<\underline{\rho}=\delta<1$, which imply that delay is costless for a type $\mathrm{H}$ but costly for a type L. Thus, it is likely that a type $\mathrm{H}$ will employ $1 \ell$ (.) or $2 \ell($.) to separate herself from a type $\mathrm{L}$ since these two sets of proposals give rise to delay with positive probabilities in equilibrium.

We derive separating and pooling equilibria in turn.

\section{Separating equilibria}

In a separating equilibrium the proposal made by a type $\mathrm{H}$ player differs from that made by a type L player. As a result, player 1's type will be revealed after the first session (i.e., $p_{1}=1$ if player 1 is a type $\mathrm{H}$, and $p_{1}=0$ if player 1 is a type $\mathrm{L}$ ). 
Our derivation proceeds mainly in two steps, as follows:

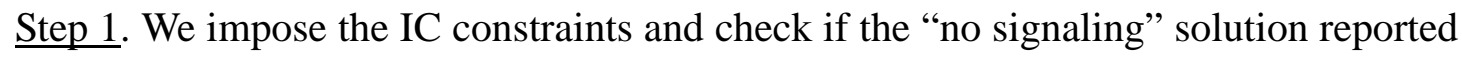
in Propositions 1-2 can be supported as a separating equilibrium. The answer will be positive if all the IC constraints are satisfied so that neither type wants to mimic the other type (the "no-envy" case). While the answer will be negative if some IC constraints are violated so that there is a type who wants to mimic the other type (the “envy" case).

Step 2. We ask in the "envy" case if the mimicked type can deviate from her "no signaling” solution and find profitable action to separate her from the type who wants to mimic her. If she can, then separating equilibria exist; if she cannot, then separating equilibria fail to exist. In the case where separating equilibria are nonempty, we characterize this set and apply the refinement of the dominance criterion to arrive at the so-called "least-cost” separating equilibrium.

\section{Pooling equilibria}

In a pooling equilibrium the proposal made by a type $\mathrm{H}$ is the same as that made by a type L. As a result, $p_{1}=1 / 2$. We consider three ranges of $\delta$ 's separately according to Lemma $1-3$, that is, $\delta>1 / 3,1 / 5<\delta<1 / 3$ and $\delta<1 / 5$. In each range, our derivation proceeds mainly in two steps.

Step 1. We derive the best of $1 h(\mathrm{HL}), 1 \ell(\mathrm{HL})$ and $2 \ell(\mathrm{HL})$, which together with the separating equilibria derived are used to pin down the possible candidates of pooling equilibria.

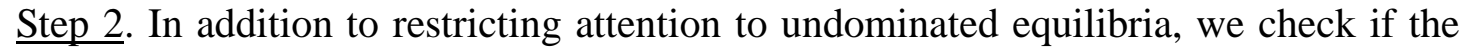
possible candidates of pooling equilibria survive under the refinement of Cho and Krep’s (1987) intuitive criterion. An equilibrium that survives the refinement of the intuitive criterion will be called an intuitive equilibrium.

The following proposition summarizes our finding:

Proposition 3. Suppose that the type of the proposer (player 1) is unknown. Then, the proposals in Session one are as follows: 
a. If $\delta>1 / 2$, there is a unique intuitive equilibrium with monotone beliefs in which both types pool on the proposal $(2 / 3,1 / 3,0)$.

b. If $1 / 3<\delta<1 / 2$, there is a continuum of intuitive equilibria with monotone beliefs in which both types pool and all proposals obey minimum winning coalitions.

c. If $\delta_{1}<\delta<1 / 3$ where $\delta_{1}=5 / 19 \approx 0.263$, the best of $2 \ell(\mathrm{H})$ and the best of $1 h(\mathrm{~L})$ constitute the unique separating equilibrium with monotone beliefs in which neither type envies the other type. All pooling equilibria with monotone beliefs are dominated by this "no envy" separating equilibrium.

d. If $\delta_{0}<\delta<\delta_{1}$ where $\delta_{0}=(23-3 \sqrt{41}) / 16 \approx 0.237$, the "least-cost" separating equilibrium and a continuum of intuitive pooling equilibria with monotone beliefs coexist. Except for the best of $1 \mathrm{~h}(\mathrm{~L})$ in the least-cost separating equilibrium, all proposals adopt oversized coalitions.

e. If $\delta<\delta_{0}$, there is a continuum of intuitive equilibria with monotone beliefs in which both types pool and all proposals adopt oversized coalitions.

Figure 1 illustrates the resulting equilibrium proposals for different values of $\delta$. We refer the reader to the proof in the Appendix for the actual formulas of the proposals.

\section{[Insert Figure 1 about here]}

The pattern of minimum winning coalitions versus oversized coalitions in Proposition 3 is similar to that in Propositions 1-2. We focus on why proposals pool or separate. Our explanation proceeds along two dimensions. First, signaling type $\mathrm{H}$ or $\mathrm{L}$, that is, is it better or worse off for a player to be regarded as a type $\mathrm{H}$ or $\mathrm{L}$ ? Second, capability and profitability of separation, that is, is it capable and profitable for a type to signal her type if being regarded as that type makes her better off?

\section{Signaling type $H$ or $L$}

In Spence-style signaling models, both $\mathrm{H}$ and L types want to be regarded as the 
$\mathrm{H}$ type, as the wage payment is monotonically increasing in the expected types. This stylized result need not hold in our context. There are two conflicting concerns involved. On the one hand, being recognized as a type $L$ ensures a coalitional offer in the second session, but this coalitional offer will be the lower $1 \ell((1 / 3) \delta)$ rather than the higher $1 h(1 / 3)$ (Lemma 1-2). On the other hand, being recognized as a type $\mathrm{H}$ ensures the higher offer $1 h$ rather than the lower offer $1 \ell$ if there is a coalitional offer in the second session, but the incidence of this coalitional offer occurs only with a 1/2 chance (Lemma 1-1). For convenience, we coin them as the "coalition" and the "offer" concerns, respectively. Whether being regarded as a type $\mathrm{H}$ rather than a type $\mathrm{L}$ makes a player better or worse off is not predetermined a priori in our model, but depends on these two conflicting concerns. In Spence-style models, there is only the "offer" concern involved and hence both the $\mathrm{H}$ and L types would like to be regarded as a type $\mathrm{H}$ a priori. The endogeneity of coalition partners in majoritarian bargaining alters this feature in a fundamental way.

Obviously, player 1 (the proposer) prefers to be regarded as a type L if $\delta>1 / 2$, whereas she prefers to be regarded as a type $\mathrm{H}$ if $\delta<1 / 2$. There will be no difference for player 1 if and only if $(1 / 3) \delta=(1 / 2)(1 / 3)$, which gives rise to the border $\delta=1 / 2$. Intuitively, when $\delta>1 / 2$ so that the value of $\delta$ is high, it is better off to be recognized as a type $L$ and to earn the sure payoff $(1 / 3) \delta$ in the second session (the "coalition" concern dominates the "offer" concern); however, when $\delta<1 / 2$ so that the value of $\delta$ is low, being recognized as a type $\mathrm{H}$ and earning the higher payoff $1 / 3$ with probability $1 / 2$ in the second session becomes an advantage (the "offer” concern dominates the "coalition” concern).

When bargaining requires the unanimous consent of all players, it is known that a patient player will fare better than an impatient player. However, as pointed out by Harrington (1990), Persson and Tabellini (2000, p. 39) and others, the opposite occurs when bargaining requires only a majority consent. This is because patience means a higher continuation value, and the higher the continuation value the higher the reservation price; therefore, the lower the probability that a player will be made a 
member of the winning coalition in majoritarian bargaining. Following this line of argument, one would expect a type L (an impatient player) would have an incentive to signal her type, while a type $\mathrm{H}$ (a patient player) would have an incentive to hide her type. Neither is necessarily true in our model. The arguments put forth by Harrington (1990) and Persson and Tabellini (2000) are mainly based on the "coalition" concern and there is no "offer" concern involved. In these majoritarian bargaining models, each player has complete information about other players' types and, therefore, there is no role for the “offer” concern à la Spence’s signaling.

\section{Capability and profitability of separation}

When $\delta>1 / 2$, a type $\mathrm{L}$ is better off being recognized as a type $\mathrm{L}$ since the “coalition" concern dominates the "offer" concern. However, while it is one thing for a type $\mathrm{L}$ to be better off being recognized as a type $\mathrm{L}$, as to whether a type $\mathrm{L}$ is capable of taking action to separate herself from a type $\mathrm{H}$ is another thing. In our model, one type of player distinguishes herself from the other type of player by exercising delay. Since delay is costly for a type $\mathrm{L}$ but costless for a type $\mathrm{H}$, there is no way for a type L to signal her type and separate herself from a type $\mathrm{H}$ via delay: every delay action taken by a type $L$ can be imitated without cost by a type $H$. It is of no surprise to find that there are no separating but pooling equilibria if $\delta>1 / 2$.

When $\delta<1 / 2$, a type $\mathrm{H}$ is better off being recognized as a type $\mathrm{H}$ since the “offer” concern dominates the "coalition” concern. Delay is costless for a type H but costly for a type $\mathrm{L}$, and hence a type $\mathrm{H}$ has the ability to take action to separate herself from a type L. However, capability does not automatically imply profitability. In the regime where $\delta>1 / 3$, both types have an intrinsic dislike of delay (Propositions 1a and 2a). This is because proposals taking the form $2 \ell($.) are too expensive as compared with those taking the form $1 h($ ) (the difference between $\bar{\rho}=1$ and $\underline{\rho}=\delta$

is relatively small) while proposals taking the form $1 \ell($.) are too risky as compared with those taking the form $1 h($.$) (the probability of failing to receive a majority$ 
support is quite high for $1 \ell()$.$) . As a result, a type H$ has no incentive to separate herself from a type L simply because delay is too costly and hence not profitable. Without a type $\mathrm{H}$ exercising delay, a type $\mathrm{L}$ can always mimic a type $\mathrm{H}$. This explains why there are no separating but pooling equilibria $1 h(\mathrm{HL})$ if $1 / 3<\delta<1 / 2$.

The intrinsic preferences for delay differ between the types in the range where $\delta_{0}<\delta<1 / 3$ : while a type $\mathrm{L}$ continues to have an intrinsic dislike of delay, a type $\mathrm{H}$ develops an intrinsic liking for delay (Propositions $1 \mathrm{~b}$ and 2a). This difference in preference makes room for a type $\mathrm{H}$ to separate herself from a type L. Indeed, if $\delta_{1}<\delta<1 / 3$ such that the value of $\delta$ is low enough for a type $\mathrm{H}$ to optimally buy two risky votes (i.e., choose the best of $2 \ell(\mathrm{H})$ ) but high enough for a type $\mathrm{L}$ to optimally buy one sure vote (i.e., choose the best of $1 h(\mathrm{~L})$ ), a type $\mathrm{L}$ will no longer envy a type $\mathrm{H}$ and try to mimic her. Intuitively, delay is costless for a type $\mathrm{H}$ but costly for a type $\mathrm{L}$ and hence although being regarded as a type $\mathrm{H}$ is better off for a type $\mathrm{L}$, it is too costly for a type $\mathrm{L}$ to mimic a type H's choice of $2 \ell(\mathrm{H})$ in this regime. This explains why the "no-envy" separating equilibrium can be sustained if $\delta_{1}<\delta<1 / 3$.

However, once the value of $\delta$ is further lowered to the range where $\delta_{0}<\delta<\delta_{1} \quad\left(\delta_{1}=5 / 19\right.$ is the border which makes a type $\mathrm{L}$ indifferent between the best of $1 h(\mathrm{~L})$ and the best of $2 \ell(\mathrm{H}))$, the price of buying two risky votes will become low enough to compensate for a type L's cost of delay. As a result, it is even profitable for a type $\mathrm{L}$ to mimic a type $\mathrm{H}$ by choosing $2 \ell(\mathrm{H})$. This excludes the “no-envy” separating equilibrium. In Spence-style signaling models, one can derive the so-called "least-cost" separating equilibrium in which a type $\mathrm{H}$ player chooses an action with the least distortion to separate herself from a type L. ${ }^{10}$ Since delay is costly for a type L but costless for a type $\mathrm{H}$, and since a type $\mathrm{H}$ has an intrinsic liking for delay but a type $\mathrm{L}$ has an intrinsic dislike of delay in this regime, a similar “least-cost” separating equilibrium does exist.

In Spence-style models, all the pooling equilibria that survive the dominance of

10 See Bolton and Dewatripont (2005, p. 109). 
the "least-cost" separating equilibrium can be broken down after refining the equilibrium set based on the intuitive criterion. In contrast, all the pooling equilibria that survive the dominance of the "least-cost" separating equilibrium in our model coexist with the "least-cost" separating equilibrium even after the refinement of the intuitive criterion. Intuitively, once it is profitable for a type $\mathrm{L}$ to bear the cost of delay by mimicking a type H's choice of $2 \ell(\mathrm{H})$, it is no longer possible for a type $\mathrm{H}$ to take delay action to distinguish herself from a type L.

When $\delta<\delta_{0}$, both types want to masquerade as the other type. The logic underlying a type $\mathrm{L}$ mimicking a type $\mathrm{H}$ remains the same as before. However, the logic underlying a type $\mathrm{H}$ mimicking a type $\mathrm{L}$ is quite different. Note that both types have an intrinsic liking for $2 \ell$ (.) in this regime (Propositions $1 \mathrm{~b}$ and $2 \mathrm{~b}$ ). Following the belief $p=(0,1,1)$ (from a rejection of $2 \ell(\mathrm{L})$ ), player 1 will always be included in the coalition formation in the second session if player 2 or 3 is recognized as being the proposer (Lemma 1-2). The intrinsic liking for $2 \ell(\mathrm{L})$ by a type $\mathrm{L}$ gives a type $\mathrm{H}$ an opportunity to enhance her payoff: a type $\mathrm{H}$ could pretend to be a type $\mathrm{L}$ to ensure a coalitional offer in the second session on the equilibrium path and then reject the offer. The rejection implies that the game would move to the third session in equilibrium, which in turn implies that it would ensure a type H player the payoff 1/3 (a type H's continuation value). This pretend-and-then-reject strategy, if available, would resolve the conflict between the "coalition" and the "offer" concern: not only does a type $\mathrm{H}$ ensure the coalitional offer in the second session but it also ensures the higher payoff $1 / 3$ by rejecting the coalitional offer.

The reasoning above is built on the existence of the belief $p=(0,1,1)$. However, as we have just argued, the very existence of the belief $p=(0,1,1)$ would induce a type $\mathrm{H}$ to mimic a type $\mathrm{L}$ and hence the pooling of a type $\mathrm{H}$ with a type $\mathrm{L}$. This indicates that the existence of a separating equilibrium to support $p=(0,1,1)$ cannot be true in the first place. In other words, there will be no separating equilibria in this regime.

As to the existence of the pooling equilibria, the reasoning is similar to that in the regime where $\delta_{0}<\delta<\delta_{1}$. A main difference is that since the price of buying two 
risky votes has become low enough in this regime, even a type $\mathrm{L}$ has an intrinsic liking for delay (Proposition 2b). This explains the basic difference between $\delta<\delta_{0}$ and $\delta_{0}<\delta<\delta_{1}$ : separating and pooling equilibria coexist if $\delta_{0}<\delta<\delta_{1}$, whereas there are only pooling equilibria if $\delta<\delta_{0}$.

\section{Beyond three sessions}

By applying the same procedure as in the three-session game, we can derive solutions for a game with any finite number of sessions. However, the derivation becomes very tedious and lengthy once the number of sessions exceeds three. Instead of repeating the same derivation procedure, we focus on exposing the logic behind the two additional features of equilibrium solutions, which arise only if the game lasts more than three sessions. We illustrate these two features with the four-session game, and touch on what would happen if the game lasts more than four sessions.

\subsection{Four-session game}

As long as all players choose the $1 h$ proposal in the penultimate session, the ex ante value of the game at the beginning of the penultimate session will equal $1 / 3$ for all players (see $A(1)$ ), which is the same as the ex ante value of the game at the beginning of the last session. In such a situation, a $T$-session game can de facto reduce to a game that lasts only $T-1$ sessions.

From Lemma 1, we see that all players will choose the $1 \mathrm{~h}$ proposal if (i) $p_{1}=1$, (ii) $p_{1}=1 / 2$ with $p=(1 / 2,1,1)$, or (iii) $p_{1}=1 / 2$ with $p=(1 / 2,1,1 / 2)$ and $\delta \geq 1 / 3$. Put differently, there are only two situations under which a game that lasts more than three sessions may not be reduced to the three-session game. First, $p_{1}=0$; second, $p_{1}=1 / 2$ with $p=(1 / 2,1,1 / 2)$ and $\delta<1 / 3$. The two additional features of equilibrium solutions mentioned above are associated with these two situations, respectively. 
The following proposition summarizes our finding for the four-session game: ${ }^{11}$

Proposition 4. Suppose that the type of the proposer (player 1) is unknown. By replacing $\delta_{0} \approx 0.237$ with $\delta_{00} \approx 0.246$ and $\delta_{1} \approx 0.263$ with $\delta_{11} \approx 0.28$, the qualitative properties of the proposals in Session one remain the same as those in Proposition 3, except that:

1. If $\delta>\delta_{2}$ where $\delta_{2}=(-3+\sqrt{37}) / 4 \approx 0.77$, the unique intuitive pooling equilibrium in Proposition $3 a$ is expanded to a continuum of intuitive pooling equilibria in which all proposals obey minimum winning coalitions.

2. If $1 / 6<\delta<1 / 3$, in addition to the equilibria in Proposition 3, there is a continuum of intuitive pooling equilibria in which all proposals obey minimum winning coalitions.

Figure 2 illustrates the resulting equilibrium proposals for different values of $\delta$.

\section{[Insert Figure 2 about here]}

Both results reported in Proposition 4 are related to the alternation of coalition partners. The first result is associated with $p_{1}=0$, and it arises because players 2 and 3 if recognized in session two choose each other as their coalition partner in the four-session game, whereas they both choose player 1 as their coalition partner in the three-session game. The second result is associated with $p_{1}=1 / 2$ with $p=(1 / 2,1,1 / 2)$ and $\delta<1 / 3$. It arises because player 1 (3) if recognized in session two chooses player 3 (1) as her coalition partner and leaves out player 2 in the four-session game, whereas she is indifferent to either choosing player 2 or player 3 (1) as her coalition partner in the three-session game.

By introducing asymmetric tie-breaking rules or heterogeneous time preferences into the finite horizon version of the BF model, Norman (2002, p. 335) observed: "the

\footnotetext{
${ }^{11}$ Since the derivation is basically the same as that in the three-session game, we do not provide the proof for the four-session game in this paper. For a sketch of the proof of Proposition 4 and other claims in this section, see the website: http://mx.nthu.edu.tw/ tstsai/
} 
player that got favorable treatment in the second period was at a disadvantage since she was too expensive to bribe in the first period.” A similar logic applies to our finding. Let us consider Proposition 4-1 first. Suppose that player 1 is known to be a type $\mathrm{L}$ in session two (i.e., $p_{1}=0$ ). Both players 2 and 3, if they are recognized, will seek player 1 as their coalition partner in the third session of the four-session game (Lemma 1-2). This implies that player 1 will have a higher continuation value and hence it becomes rather expensive to include player 1 in the coalition in the second session of the four-session game. Indeed, we find that once $\delta>\delta_{2} \approx 0.77$ so that the ensured payoff for player 1 (i.e., (1/3) $\delta$ ) in the third session becomes high enough, it will be better for players 2 and 3 to choose each other as their coalition partner and leave out player 1 in the second session of the four-session game if they are recognized. Player 1 is left out of the coalition even though she is known to be a type L. This gives rise to an incentive for a type- $\mathrm{L}$ player 1 to signal $\mathrm{H}$ rather than $\mathrm{L}$, a result opposite to that found in the three-session game.

Next, let us consider Proposition 4-2. This result is attributed to the belief $p=(1 / 2,1,1 / 2)$. In the three-session game with $p=(1 / 2,1,1 / 2)$, player 1 is indifferent toward choosing player 2 with $p_{2}=1$ or player 3 with $p_{3}=1 / 2$ as her coalition partner. This is because the ex ante value of the game at the beginning of the third session equals $1 / 3$ for all players in the three-session game. In the four-session game with $p=(1 / 2,1,1 / 2)$, player 1 remains indifferent toward players 2 and 3 if $\delta \geq 1 / 3$, but this is no longer true if $\delta<1 / 3$. Given $p=(1 / 2,1,1 / 2)$, let $q=\left(q_{1}, q_{2}, q_{3}\right)$ with $q_{j}$ denote the common posterior at the beginning of the third session that players other than $j$ attach to the possibility that player $j$ is a type $\mathrm{H}$. Suppose that player 1 is recognized to become the proposer in the second session of the four-session game. Then, choosing player 2 as her coalition partner will lead to $q=(1 / 2,1,1 / 2)$, while choosing player 3 as her coalition partner will lead to $q=(1 / 2,1,1)$. According to Lemma 1 (with the replacement of $p$ with $q$ ), players' behavior facing $q=(1 / 2,1,1 / 2)$ can deviate from that facing $q=(1 / 2,1,1)$ if 
$\delta<1 / 3$. In contrast, players' behavior facing $q=(1 / 2,1,1 / 2)$ remains the same as that facing $q=(1 / 2,1,1)$ in the three-session game. This difference explains why there is no correspondence of Proposition 4-2 in Proposition 3.

Note that the equilibrium proposals in Proposition 4-2 take the form of $1 h(\mathrm{HL})$. This is because, with $p=(1 / 2,1,1 / 2)$ and $\delta<1 / 3$, players 1 and 3 will prefer to choose each other as their coalition partner and leave player 2 out if they are recognized to become the proposer in the second session of the four-session game. This second-session coalition formation, which excludes player 2, lowers player 2's continuation value and thus makes it rather cheap for player 1 to buy player 2's sure vote in the first session of the game. A separating equilibrium arises in our model mainly because a type $\mathrm{H}$ is more tolerant toward delay than a type L. However, this preference difference in delay tolerance would be overwhelmed by the rather low price to buy the sure vote from player 2 if the game lasts four sessions. This explains why the new form of the pooling equilibria $1 h(\mathrm{HL})$ can coexist with the “no-envy” and the "least-cost” separating proposals in the four-session game.

\subsection{More than four sessions}

An important implication of Norman's observation is that the identity of the players that are included in the majoritarian coalition may vary across periods. This leads Norman to find that proposals do not converge in his model. A similar outcome arises in our model. Consider the case where $p_{1}=0$. Whenever players 2 and 3 seek each other as their coalition partner and leave player 1 out in session $t+1$, it becomes rather cheap to include player 1 in the coalition in session $t$. Once both players 2 and 3 seek player 1 as their coalition partner and leave each other out in session $t$, it becomes rather expensive to include player 1 in the coalition in session $t-1$ and, as a result, players 2 and 3 are once again better off seeking each other as their coalition partner. This alternation of coalition partners could continue, resulting 
in a non-stationary outcome. ${ }^{12}$ An interesting outcome that emerges is that whether being regarded as a type $\mathrm{H}$ rather than a type $\mathrm{L}$ makes a player better or worse off depends not only on the value of $\delta$, but also on the number of sessions that a game lasts. For example, if $\delta>\delta_{2} \approx 0.77$, player 1 would like to be regarded as a type L in the three-session game, whereas she would like to be regarded as a type $\mathrm{H}$ in the four-session game. This difference in preference of course stems from the alternation of coalition partners.

Next, consider the case where $p=(1 / 2,1,1 / 2)$ and $\delta<1 / 3$. Whenever players 1 and 3 seek each other as their coalition partner and leave player 2 out in the second session of the four-session game, it becomes rather cheap to include player 2 in the coalition in the first session. However, once player 1 seeks player 2 as the coalition partner in the second session of the five-session game, it becomes too expensive to include player 2 in the coalition in the first session. This alternation continues, resulting in a non-stationary outcome in which proposals similar to those in Proposition 4-2 will arise if the game lasts even number of sessions but will not arise if the game lasts odd number of sessions. In other words, by confining the analysis to the regime where $\delta<1 / 3$, the first-session proposals will resemble those of Proposition 3 if the game lasts odd sessions, but they will resemble those of Proposition 4 if the game lasts even sessions. The rise of the new form of the $1 h(\mathrm{HL})$ pooling proposal in the even-session game suggests that it becomes harder for players to exercise separation as compared to the odd-session game.

\section{Conclusion}

This paper extends Baron and Ferejohn’s (1989) majoritarian bargaining game to a world with incomplete information and, in particular, it completely characterizes the

\footnotetext{
${ }^{12}$ Whether the alternation of coalition partners arises or not obviously depends on the value of $\delta$ in our model. However, its occurrence seems to lack regularity. For example, it arises in the four-session game if $\delta>0.77$, while it arises in the five-session game if $0.69<\delta<0.71$ or $\delta>0.92$. Despite its irregularity, it can be shown that in the face of $p_{1}=0$, both players 2 and 3 will always choose player 1 as their coalition partner if $\delta \leq 1 / 2$, and hence the alternation of coalition partners or the non-stationary outcome associated with $p_{1}=0$ can occur only if $\delta>1 / 2$.
} 
pure-strategy equilibrium of the game for the three-player-three-session version. The versions that we have analyzed are admittedly specific and far from general. Nevertheless, they allow us to gain insights into majoritarian bargaining with incomplete information and, more importantly, the resulting outcomes do suggest some qualitatively significant differences from that with complete information. As mentioned in the Introduction, the Baron-Ferejohn model has been applied widely to a variety of political issues. It would be interesting to know how the results obtained in these applied works would be altered when the information assumption is relaxed from complete to incomplete.

The BF model belongs to the "offer” type of majoritarian bargaining game since the way of dividing the dollar is initiated by the proposer's making a specific distributive offer to other members of the game. The way of dividing the dollar with majority rule may also take the form of the "demand" type in which players take turns in making their own demands for a share of the dollar; see, for example, Frechette et al. (2005). As we have shown, introducing private information on the discount factor to the BF model alters equilibrium outcomes significantly; in particular, oversized coalitions arise and delay emerges. It is conceivable that introducing private information on the discount factor to the "demand" type of majoritarian bargaining may not have the same impact. If so, the implication of this is a caveat: the observation of oversized coalitions or delay in reality can be attributed to the existence of private information only if the BF model is the right one to use in modeling majoritarian bargaining.

\section{Appendix}

Remember that we make the monotonicity assumption about $1 h($.$) with regard to$ its off-the-equilibrium-path beliefs, and that we only report the case where the responder indexed with a lower subscript is chosen as the coalition partner ex post whenever a proposer is indifferent over which of her two responders to include in the coalition. 


\section{Proof of Proposition 1}

By assumption, $p_{1}=1$. The posteriors after a rejection of $2 \ell(\mathrm{H}), 1 \ell(\mathrm{H})$ and 1h(H) are, respectively: $p=(1,1,1), \quad p=(1,1,1 / 2)$, and $p=(1,1,1 / 2)$. According to Lemma 1-1,

$$
v_{1}=v_{2}=v_{3}=(1 / 3)(1-(1 / 3))+(2 / 3)(1 / 2)(1 / 3)=1 / 3 \text {. }
$$

Put in words: after the rejection, each player has a $1 / 3$ chance of becoming the proposer in the second session; once a player becomes the proposer, she adopts the $1 \mathrm{~h}$ proposal with a symmetric tie-breaking.

(A1) implies:

The best of $2 \ell(\mathrm{H}), 1 \ell(\mathrm{H})$ and $1 h(\mathrm{H})$ are, respectively

$\left(1-\delta\left(v_{2}+v_{3}\right), \delta v_{2}, \delta v_{3}\right)=(1-(2 / 3) \delta,(1 / 3) \delta,(1 / 3) \delta) ;$

$\left(1-\delta v_{2}, \delta v_{2}, 0\right)=(1-(1 / 3) \delta,(1 / 3) \delta, 0)$

$\left(1-v_{2}, v_{2}, 0\right)=(1-(1 / 3), 1 / 3,0)$.

The expected payoffs from choosing these best proposals equal, respectively

$$
\begin{aligned}
& (3 / 4)\left[1-\delta\left(v_{2}+v_{3}\right)\right]+(1 / 4) v_{1}=5 / 6-(1 / 2) \delta ; \\
& (1 / 2)\left[1-\delta v_{2}\right]+(1 / 2) v_{1}=2 / 3-(1 / 6) \delta ; \\
& 1-v_{2}=2 / 3
\end{aligned}
$$

With the common knowledge that both $\mathrm{H}$ and $\mathrm{L}$ types are equally likely a priori, player 1 faces four possible types of responders in combination (i.e., HH, HL, LH and LL) with equal probability. Thus, the probability of failing to receive a majority support is $1 / 4$ if the $2 \ell(\mathrm{H})$ proposals are chosen, $1 / 2$ if the $1 \ell(\mathrm{H})$ proposals are chosen, and zero if the $1 h(\mathrm{H})$ proposals are chosen. Since player 1 is a type H, her continuation value equals $v_{1}=1 / 3$ if a proposal fails to receive a majority support so that delay occurs.

It is clear from (A2) that, regardless of the value of $\delta$, the best of $1 h(\mathrm{H})$ strictly dominates the best of $1 \ell(\mathrm{H})$. As a result, we only need to make a comparison 
between $1 h(\mathrm{H})$ and $2 \ell(\mathrm{H})$. It can be calculated that the best of $2 \ell(\mathrm{H})$ will be chosen over the best of $1 h(\mathrm{H})$ if and only if

$$
\delta<1 / 3 \text {. }
$$

Q.E.D.

\section{Proof of Proposition 2}

By assumption, $p_{1}=0$. The posteriors after a rejection of $2 \ell(\mathrm{L}), 1 \ell(\mathrm{L})$ and $1 h(\mathrm{~L})$ are, respectively: $p=(0,1,1), \quad p=(0,1,1 / 2)$, and $p=(0,1,1 / 2)$. According to Lemma 1-2,

$$
\begin{aligned}
& v_{1}=(1 / 3)(1-(1 / 3))+(2 / 3)(1 / 3) \delta=2 / 9+(2 / 9) \delta \\
& v_{2}=v_{3}=(1 / 3)(1-(1 / 3) \delta)+(1 / 3)(1 / 2)(1 / 3)=7 / 18-(1 / 9) \delta .
\end{aligned}
$$

Put in words: after the rejection, each player has a $1 / 3$ chance of being recognized as the proposer in the second session; once recognized, player 1 adopts the $1 h$ proposal with a symmetric tie-breaking, while both players 2 and 3 adopt the $1 \ell$ proposal and choose player 1 as their coalition partner.

(A4) implies:

The best of $2 \ell(\mathrm{L}), 1 \ell(\mathrm{L})$ and $1 h(\mathrm{~L})$ are, respectively

$$
\begin{aligned}
& \left(1-\delta\left(v_{2}+v_{3}\right), \delta v_{2}, \delta v_{3}\right)=\left(1-(7 / 9) \delta+(2 / 9) \delta^{2},(7 / 18) \delta-(1 / 9) \delta^{2},(7 / 18) \delta-(1 / 9) \delta^{2}\right) ; \\
& \left(1-\delta v_{2}, \delta v_{2}, 0\right)=\left(1-(7 / 18) \delta+(1 / 9) \delta^{2},(7 / 18) \delta-(1 / 9) \delta^{2}, 0\right) \\
& \left(1-v_{2}, \quad v_{2}, 0\right)=(11 / 18+(1 / 9) \delta, 7 / 18-(1 / 9) \delta, 0)
\end{aligned}
$$

The expected payoffs from choosing these best proposals equal, respectively

$$
\begin{aligned}
& (3 / 4)\left[1-\delta\left(v_{2}+v_{3}\right)\right]+(1 / 4) \delta v_{1}=3 / 4-(19 / 36) \delta+(2 / 9) \delta^{2} \\
& (1 / 2)\left[1-\delta v_{2}\right]+(1 / 2) \delta v_{1}=(1 / 2)-(1 / 12) \delta+(1 / 6) \delta^{2} \\
& 1-v_{2}=11 / 18+(1 / 9) \delta
\end{aligned}
$$

Since player 1 is a type $\mathrm{L}$, her continuation value equals $\delta v_{1}=(2 / 9) \delta+(2 / 9) \delta^{2}$ if a proposal fails to receive a majority support so that delay occurs.

Regardless of the value of $\delta$, the best of $1 h(\mathrm{~L})$ strictly dominates the best of 
$1 \ell(\mathrm{L})$. As a result, we only need to make a comparison between $1 h(\mathrm{H})$ and $2 \ell(\mathrm{H})$. It can be calculated that the best of $2 \ell(\mathrm{L})$ will be chosen over the best of $1 h(\mathrm{~L})$ if and only if

$$
\delta<\delta_{0}=\left[23-3(41)^{1 / 2}\right] / 16 \approx 0.237
$$

Q.E.D.

\section{Proof of Proposition 3}

We derive separating and pooling equilibria in turn.

\section{Separating equilibria}

A separating equilibrium requires that neither a type $\mathrm{H}$ nor $\mathrm{L}$ have an incentive to masquerade as the other type. We first check to see whether the "no signaling" solution of Propositions 1-2 can be supported as a separating equilibrium (the “no-envy” case).

\section{Step 1. "No-envy" case}

We examine the three regimes stated in Propositions 1-2 in turn.

(I) $\delta>1 / 3$

The IC constraints for this regime require that a type $\mathrm{H}$ who chooses the best of $1 h(\mathrm{H})$ has no incentive to masquerade as a type $\mathrm{L}$ who chooses the best of $1 h(\mathrm{~L})$, viz. $2 / 3 \geq 1-v_{2}$;

and that a type $\mathrm{L}$ who chooses the best of $1 h(\mathrm{~L})$ has no incentive to masquerade as a type $\mathrm{H}$ who chooses the best of $1 h(\mathrm{H})$, viz.

$1-v_{2} \geq 2 / 3$.

where $v_{2}$ follows (A4-2) since a rejection of $1 h(\mathrm{~L})$ implies $p=(0,1,1 / 2)$.

It can be checked that the IC constraint (A7-1-1) is violated if $\delta>1 / 2$, while the IC constraint (A7-1-2) is violated if $\delta<1 / 2$. In other words, a type $\mathrm{H}$ has an incentive to masquerade as a type $\mathrm{L}$ if $\delta>1 / 2$, while a type $\mathrm{L}$ has an incentive to 
masquerade as a type $\mathrm{H}$ if $\delta<1 / 2$. The "no signaling” solution, the best of $1 h(\mathrm{H})$ and $1 h(\mathrm{~L})$, cannot be supported as a separating equilibrium in this regime.

(II) $\delta<\delta_{0} \approx 0.237$

The IC constraints for this regime require that a type $\mathrm{H}$ who chooses the best of $2 \ell(\mathrm{H})$ has no incentive to masquerade as a type $\mathrm{L}$ who chooses the best of $2 \ell(\mathrm{L})$, viz.

$$
(3 / 4)(1-(2 / 3) \delta)+(1 / 4)(1 / 3) \geq(3 / 4)\left(1-\delta\left(v_{2}+v_{3}\right)\right)+(1 / 4) V_{1} ;
$$

and that a type $\mathrm{L}$ who chooses the best of $2 \ell(\mathrm{L})$ has no incentive to masquerade as a type $H$ who chooses the best of $2 \ell(H)$, viz.

$$
(3 / 4)\left(1-\delta\left(v_{2}+v_{3}\right)\right)+(1 / 4) \delta v_{1} \geq(3 / 4)(1-(2 / 3) \delta)+(1 / 4)(1 / 3) \delta
$$

where $V_{1}=4 / 9$, and $v_{1}, v_{2}$ and $v_{3}$ follow (A4) since a rejection of $2 \ell(\mathrm{L})$ implies that $p=(0,1,1)$.

Note that $V_{1}=4 / 9>v_{1}=2 / 9+(2 / 9) \delta$ in this regime. If the best of $2 \ell(\mathrm{L})$ fails to receive a majority support in the first session, it will give rise to the posterior belief $p=(0,1,1)$ and hence players 2 and 3 will make the offer $1 \ell$ to player 1 if either of them becomes the proposer in the second session (Lemma 1-2). However, sequential rationality dictates that player 1 of a type $H$, who mimics a type $L$ by choosing the best of $2 \ell(\mathrm{L})$ in the first session, will reject the $1 \ell$ offer in the second session. The rejection moves the game to the third session, which ensures the payoff $1 / 3$ for player 1 of a type $H$ (her continuation value). This leads to $V_{1}=1 / 3 \cdot(2 / 3)+2 / 3 \cdot(1 / 3)=4 / 9$ in (A7-2-1).

It can be checked that both (A7-2-1) and (A7-2-2) are violated so that both types have an incentive to masquerade as the other type. The "no signaling" solution, the best of $2 \ell(\mathrm{H})$ and $2 \ell(\mathrm{L})$, cannot be supported as a separating equilibrium in this regime.

(III) $\delta_{0}<\delta<1 / 3$

The IC constraints for this regime require that a type $\mathrm{H}$ who chooses the best of 
$2 \ell(\mathrm{H})$ has no incentive to masquerade as a type $\mathrm{L}$ who chooses the best of $1 h(\mathrm{~L})$, viz. $(3 / 4)(1-(2 / 3) \delta)+(1 / 4)(1 / 3) \geq 1-v_{2}$;

and that a type $\mathrm{L}$ who chooses the best of $1 h(\mathrm{~L})$ has no incentive to masquerade as a type $H$ who chooses the best of $2 \ell(\mathrm{H})$, viz.

$$
1-v_{2} \geq(3 / 4)(1-(2 / 3) \delta)+(1 / 4)(1 / 3) \delta
$$

where $v_{2}$ follows (A4-2) since a rejection of $1 h(\mathrm{~L})$ implies $p=(0,1,1 / 2)$.

Let $\delta_{1}=5 / 19 \approx 0.263$, which satisfies the equality part of (A7-3-2). Then it can be checked that both IC constraints (A7-3-1)-(A7-3-2) are satisfied if $\delta_{1}<\delta<1 / 3$, while the IC constraint (A7-3-2) is violated if $\delta_{0}<\delta<\delta_{1}$. In other words, the "no signaling" solution, the best of $2 \ell(\mathrm{H})$ and $1 h(\mathrm{~L})$, can be supported as a separating equilibrium if $\delta_{1}<\delta<1 / 3$; but it cannot be supported as a separating equilibrium if $\delta_{0}<\delta<\delta_{1}$.

Step 2. "Envy" case

Separating equilibria with envy exist if a type can deviate from her "no signaling" solution and find profitable ways to separate from the type who wants to mimic her. We respectively examine the four regimes under which "no-envy" separating equilibria fail to exist in Step 1.

(I) $\delta>1 / 2$

In this regime a type $\mathrm{H}$ has an incentive to masquerade as a type $\mathrm{L}$ under the "no signaling” solution. Let us fix the "no signaling” proposal of a type H (i.e., the best of $1 h(\mathrm{H})$ ), which yields the payoff that a type $\mathrm{H}$ can at least guarantee herself in any separating equilibrium. We check to see whether a type L can find profitable ways to separate herself from a type $\mathrm{H}$. There are three possibilities:

(i) A type $\mathrm{L}$ seeks solutions in the set of $1 h(\mathrm{~L})$ with $x^{1}(1)=\left(1-x_{2}^{1}(1), x_{2}^{1}(1), 0\right)$ and $x_{2}^{1}(1) \geq 7 / 18-(1 / 9) \delta$, such that it is not profitable for a type $\mathrm{H}$ to mimic, viz.

$2 / 3 \geq 1-x_{2}^{1}(1)$

but profitable for a type L to separate, viz. 


$$
1-x_{2}^{1}(1)>2 / 3
$$

However, there exist no such solutions that satisfy both inequalities above in this regime.

(ii) A type $\mathrm{L}$ seeks solutions in the set of $1 \ell(\mathrm{L})$ with $x^{1}(1)=\left(1-x_{2}^{1}(1), x_{2}^{1}(1), 0\right)$ and $x_{2}^{1}(1) \geq(7 / 18) \delta-(1 / 9) \delta^{2}$ such that it is not profitable for a type $\mathrm{H}$ to mimic, viz.

$$
2 / 3 \geq(1 / 2)\left(1-x_{2}^{1}(1)\right)+(1 / 2) \mathrm{V}_{1} ;
$$

but profitable for a type L to separate, viz.

$$
(1 / 2)\left(1-x_{2}^{1}(1)\right)+(1 / 2) \delta v_{1}>2 / 3,
$$

where $V_{1}=4 / 9$, and $v_{1}$ follows (A4- 1 ) since a rejection of $1 \ell(\mathrm{L})$ implies that $p=(0,1,1 / 2)$. However, there exist no such solutions that satisfy both inequalities above in this regime.

(iii) A type $\mathrm{L}$ seeks solutions in the set of $2 \ell(\mathrm{L})$ with $x^{1}(1)=\left(1-x_{2}^{1}(1), x_{2}^{1}(1), x_{3}^{1}(1)\right)$ and $x_{2}^{1}(1), \quad x_{3}^{1}(1) \geq(7 / 18) \delta-(1 / 9) \delta^{2}$ such that it is not profitable for a type $\mathrm{H}$ to mimic, viz.

$$
2 / 3 \geq(3 / 4)\left(1-x_{2}^{1}(1)-x_{3}^{1}(1)\right)+(1 / 4) \mathrm{V}_{1} ;
$$

but it is profitable for a type L to separate, viz.

$$
(3 / 4)\left(1-x_{2}^{1}(1)-x_{3}^{1}(1)\right)+(1 / 4) \delta v_{1}>2 / 3,
$$

where $V_{1}=4 / 9$, and $v_{1}$ follows (A4- 1 ) since a rejection of $2 \ell$ (L) implies that $p=(0,1,1)$. However, there exist no such solutions that satisfy both inequalities above in this regime.

(II) $1 / 3<\delta<1 / 2$

In this regime a type $\mathrm{L}$ has an incentive to masquerade as a type $\mathrm{H}$ under the "no signaling” solution. The proof is much like the proof of (I).

(III) $\delta<\delta_{0}$ 
In this regime both types have an incentive to masquerade as the other type under the "no signaling" solution. Let us fix the "no signaling” proposal of a type L (i.e., the best of $2 \ell(\mathrm{L}))$, which yields the payoff that a type $\mathrm{L}$ can at least guarantee herself in any separating equilibrium. A rejection of $2 \ell(\mathrm{L})$ implies that $p=(0,1,1)$, which in turn implies that in the second session both players 2 and 3 will choose $1 \ell$ with player 1 as their coalition partner according to Lemma 1-2. A type $H$ could choose $2 \ell(\mathrm{L})$ and pretend to be a type $\mathrm{L}$ to ensure a coalitional offer in the second session on the equilibrium path and then reject the offer. The rejection implies that the game would move to the third session in equilibrium, which in turn implies that it would ensure a type $H$ the payoff $1 / 3$. This would give a type $H$ a higher payoff than choosing $2 \ell(\mathrm{H})$. In other words, a type $\mathrm{H}$ would mimic a type $\mathrm{L}$ rather than find ways to separate herself from a type L.

(IV) $\delta_{0}<\delta<\delta_{1}$

In this regime a type $\mathrm{L}$ has an incentive to masquerade as a type $\mathrm{H}$ under the "no signaling” solution. Let us fix the "no signaling” proposal of a type L (i.e., the best of $1 h(\mathrm{~L})$ ), which yields the payoff that a type L can at least guarantee herself in any separating equilibrium. The proof is much like the proof of (I), except for the following case. A type $\mathrm{H}$ seeks solutions in the set of $2 \ell(\mathrm{H})$ with $x^{1}(1)=\left(1-x_{2}^{1}(1), \quad x_{2}^{1}(1), \quad x_{3}^{1}(1)\right)$ and $x_{2}^{1}(1), \quad x_{3}^{1}(1) \geq(1 / 3) \delta$ such that it is not profitable for a type L to mimic, viz.

$$
11 / 18+(1 / 9) \delta \geq(3 / 4)\left(1-x_{2}^{1}(1)-x_{3}^{1}(1)\right)+(1 / 4) \delta v_{1} ;
$$

but profitable for a type $\mathrm{H}$ to separate, viz.

$$
(3 / 4)\left(1-x_{2}^{1}(1)-x_{3}^{1}(1)\right)+(1 / 4) v_{1}>11 / 18+(1 / 9) \delta,
$$

where $v_{1}$ follows (A1), since a rejection of $2 \ell(\mathrm{H})$ implies that $p=(1,1,1)$.

The above two inequalities imply

$$
5 / 27-(1 / 27) \delta \leq x_{2}^{1}(1)+x_{3}^{1}(1)<8 / 27-(4 / 27) \delta
$$


The $2 \ell(\mathrm{H})$ proposals that meet both $x_{2}^{1}(1), x_{3}^{1}(1) \geq(1 / 3) \delta$ and (A8) do exist in this regime. Thus, separating equilibria are characterized by the feature that a type $\mathrm{L}$ proposes the best of $1 h(\mathrm{~L})$, while a type $\mathrm{H}$ proposes the $2 \ell(\mathrm{H})$ proposals that satisfy (A8) with $x_{2}^{1}(1), \quad x_{3}^{1}(1) \geq(1 / 3) \delta$.

Applying the refinement of the dominance criterion and hence ascribing zero weight to the mimicking type in the set of the separating equilibria allows the mimicked type to choose the separating equilibrium she prefers most. This leads to the so-called "least-cost" separating equilibrium in which a type L proposes the best of $1 h(\mathrm{~L})$, while a type $\mathrm{H}$ makes a proposal merely satisfying the first equality part of (A8), that is,

$$
x^{1}(1)=(22 / 27+(1 / 27) \delta, 5 / 54-(1 / 54) \delta, 5 / 54-(1 / 54) \delta) .
$$

\section{Pooling equilibria}

The posteriors after a rejection of $2 \ell(\mathrm{HL}), 1 \ell(\mathrm{HL})$ and $1 h(\mathrm{HL})$ are, respectively: $p=(1 / 2,1,1), \quad p=(1 / 2,1,1 / 2)$, and $p=(1 / 2,1,1 / 2)$. According to Lemma 1-3, there are three ranges of $\delta$ 's to consider, that is, $\delta>1 / 3$, $1 / 5<\delta<1 / 3$, and $\delta<1 / 5$. The logic of the proof remains more or less the same across different ranges. We report the derived results and provide a proof only for the case where $\delta>1 / 3$.

(I) $\delta>1 / 3$

According to Lemmas 1-1 and 1-3, all players will adopt the $1 \mathrm{~h}$ proposal in the second session. Thus,

$v_{1}=v_{2}=v_{3}=(1 / 3)(1-(1 / 3))+(2 / 3)(1 / 2)(1 / 3)=1 / 3$

which implies:

The best of $2 \ell(\mathrm{HL}), 1 \ell(\mathrm{HL})$ and $1 h(\mathrm{HL})$ are, respectively $(1-(2 / 3) \delta,(1 / 3) \delta,(1 / 3) \delta)$; 
$(1-(1 / 3) \delta,(1 / 3) \delta, 0)$;

$(1-(1 / 3),(1 / 3), 0)$.

There are two sub-regimes to consider.

(I-1) $\delta>1 / 2$

The mimicking player is a type $\mathrm{H}$ in this regime. A type $\mathrm{H}$ can reveal herself and guarantee obtaining the payoff $2 / 3$ by proposing the best of $1 h(\mathrm{H})$. Thus, to prevent a deviation to the best of $1 h(\mathrm{H})$, any pooling equilibrium must give a type $\mathrm{H}$ at least the payoff $2 / 3$. For a type $\mathrm{H}$, the best of $2 \ell(\mathrm{HL})$ yields the expected payoff $(3 / 4)(1-(2 / 3) \delta)+(1 / 4)(1 / 3)=5 / 6-(1 / 2) \delta$, while the best of $1 \ell(\mathrm{HL})$ yields the expected payoff $(1 / 2)(1-(1 / 3) \delta)+(1 / 2)(1 / 3)=2 / 3-(1 / 6) \delta$. Both are strictly less than $2 / 3$ with $\delta>1 / 2$. This leads to the conclusion that the best of $1 h(\mathrm{HL})$, which yields player 1 the payoff $2 / 3$, is the only potential candidate for the pooling equilibrium.

We check if the best of $1 h(\mathrm{HL})$ survives after applying the refinement of the intuitive criterion. This refinement requires us to examine whether there exists a proposal $x^{1}(1)$ deviating from the best of $1 h(\mathrm{HL})$ such that if the deviation is regarded as originating from a type $\mathrm{L}$, a type $\mathrm{H}$ would be worse off mimicking a type L while a type L would be better off separating. There are three possibilities:

(i) Deviations are from the set of $1 h(\mathrm{~L})$ with $x^{1}(1)=\left(1-x_{2}^{1}(1), x_{2}^{1}(1), 0\right)$ and $x_{2}^{1}(1) \geq 7 / 18-(1 / 9) \delta$ such that a type $\mathrm{H}$ is worse off, viz.

$$
2 / 3>1-x_{2}^{1}(1) \text {; }
$$

but a type $\mathrm{L}$ is better off, viz.

$$
1-x_{2}^{1}(1)>2 / 3 \text {. }
$$

However, there exist no such proposals that satisfy both inequalities above in this regime.

(ii) Deviations are from the set of $1 \ell(\mathrm{L})$ with $x^{1}(1)=\left(1-x_{2}^{1}(1), x_{2}^{1}(1), 0\right)$ and 
$x_{2}^{1}(1) \geq(7 / 18) \delta-(1 / 9) \delta^{2}$ such that a type $\mathrm{H}$ is worse off , viz.

$$
2 / 3>(1 / 2)\left(1-x_{2}^{1}(1)\right)+(1 / 2) \mathrm{V}_{1}
$$

but a type $L$ is better off, viz.

$$
(1 / 2)\left(1-x_{2}^{1}(1)\right)+(1 / 2) \delta v_{1}>2 / 3,
$$

where $V_{1}=4 / 9$, and $v_{1}$ follows (A4- 1 ) since a rejection of $1 \ell(\mathrm{L})$ implies that $p=(0,1,1 / 2)$. However, there exist no such proposals that satisfy both inequalities above in this regime.

(iii) Deviations are from the set of $2 \ell(\mathrm{L})$ with $x^{1}(1)=\left(1-x_{2}^{1}(1), x_{2}^{1}(1), x_{3}^{1}(1)\right)$ and $x_{2}^{1}(1), \quad x_{3}^{1}(1) \geq(7 / 18) \delta-(1 / 9) \delta^{2}$ such that it is worse off for a type H, viz.

$$
2 / 3 \geq(3 / 4)\left(1-x_{2}^{1}(1)-x_{3}^{1}(1)\right)+(1 / 4) \mathrm{V}_{1}
$$

but better off for a type L, viz.

$$
(3 / 4)\left(1-x_{2}^{1}(1)-x_{3}^{1}(1)\right)+(1 / 4) \delta v_{1}>2 / 3,
$$

where $V_{1}=4 / 9$, and $v_{1}$ follows (A4-1) since a rejection of $2 \ell(\mathrm{L})$ implies that $p=(0,1,1)$. However, there exist no such proposals that satisfy both inequalities above in this regime.

\section{(I-2) $1 / 3<\delta<1 / 2$}

The mimicking player is a type $\mathrm{L}$ in this regime. The proof is much like the proof of (I-1).

To sum up, the best of $1 h(\mathrm{HL})$ is the unique intuitive pooling equilibrium if $\delta>1 / 2$. Those equilibria of $1 h(\mathrm{HL})$ with $1 / 3 \leq x_{2}^{1}(1) \leq 7 / 18-(1 / 9) \delta$ are the intuitive pooling equilibria if $1 / 3<\delta<1 / 2$.

(II) $1 / 5<\delta<1 / 3$

All pooling equilibria are dominated by the "no envy" separating equilibrium if $\delta_{1}<\delta<1 / 3$. Those of $2 \ell$ (HL) with $(1 / 3) \delta \leq x_{2}^{1}(1)=x_{3}^{1}(1) \leq 5 / 54-(1 / 54) \delta$ 
are the intuitive pooling equilibria if $\delta_{0}<\delta<\delta_{1}$. Those of $2 \ell$ (HL) with $(1 / 3) \delta \leq x_{2}^{1}(1)=x_{3}^{1}(1) \leq(7 / 18) \delta-(1 / 9) \delta^{2}$ are the intuitive pooling equilibria if $1 / 5<\delta<\delta_{0}$.

(III) $\delta<1 / 5$

Those equilibria of $2 \ell$ (HL) with $(1 / 3) \delta \leq x_{2}^{1}(1)=x_{3}^{1}(1) \leq(7 / 18) \delta-(1 / 9) \delta^{2}$ are the intuitive pooling equilibria if $\delta<1 / 5$.

Q.E.D. 


\section{References}

Ausubel, L.M., Cramton, P., Deneckere, R.J., 2003. Bargaining with incomplete information. In: Aumann, R.J., Hart, S. (Eds.), Handbook of Game Theory with Economic Applications, Vol. 3. North-Holland, Amsterdam.

Baron, D., Ferejohn, J., 1989. Bargaining in legislatures. American Political Science Review 83, 1181-1206.

Bolton, P., Dewatripont, M., 2005. Contract Theory. MIT Press, Cambridge.

Cho, I.-K., Kreps, D., 1987. Signaling games and stable equilibria. Quarterly Journal of Economics 102, 179-221.

Frechette, G., Kagel, J.H., Morelli, M., 2005. Behavioral identification in coalitional bargaining: An experimental analysis of demand bargaining and alternating offers. Econometrica 73, 1893-1937.

Harrington, J., 1990. The role of risk preferences in bargaining when acceptance of a proposal requires less than unanimous approval. Journal of Risk and Uncertainty 3, 135-154.

Mas-Colell, A., Whinston, M., Green, J., 1995. Microeconomic Theory. Oxford University Press, Oxford.

Norman, P., 2002. Legislative bargaining and coalition formation. Journal of Economic Theory 102, 322-353.

Persson, T., Tabellini, G., 2000. Political Economics: Explaining Economic Policy. MIT Press, Cambridge.

Riker, W., 1962. The Theory of Political Coalitions. Yale University Press, New Haven, CT.

Rubinstein, A., 1982., Perfect equilibrium in a bargaining model. Econometrica 50, 97-109.

Rubinstein, A., 1985. Choice of conjectures in a bargaining game. In: Roth, A. (Ed.), Game Theoretic Models of Bargaining. Cambridge University Press, Cambridge.

Sinclair, B., 1995. House special rules and the institutional design controversy. In: Shepsle, K., Weingast, B. (Eds.), Positive Theories of Congressional Institutions. University of Michigan Press, Ann Arbor.

Stahl, I., 1972. Bargaining Theory. Economic Research Unit, Stockholm. 


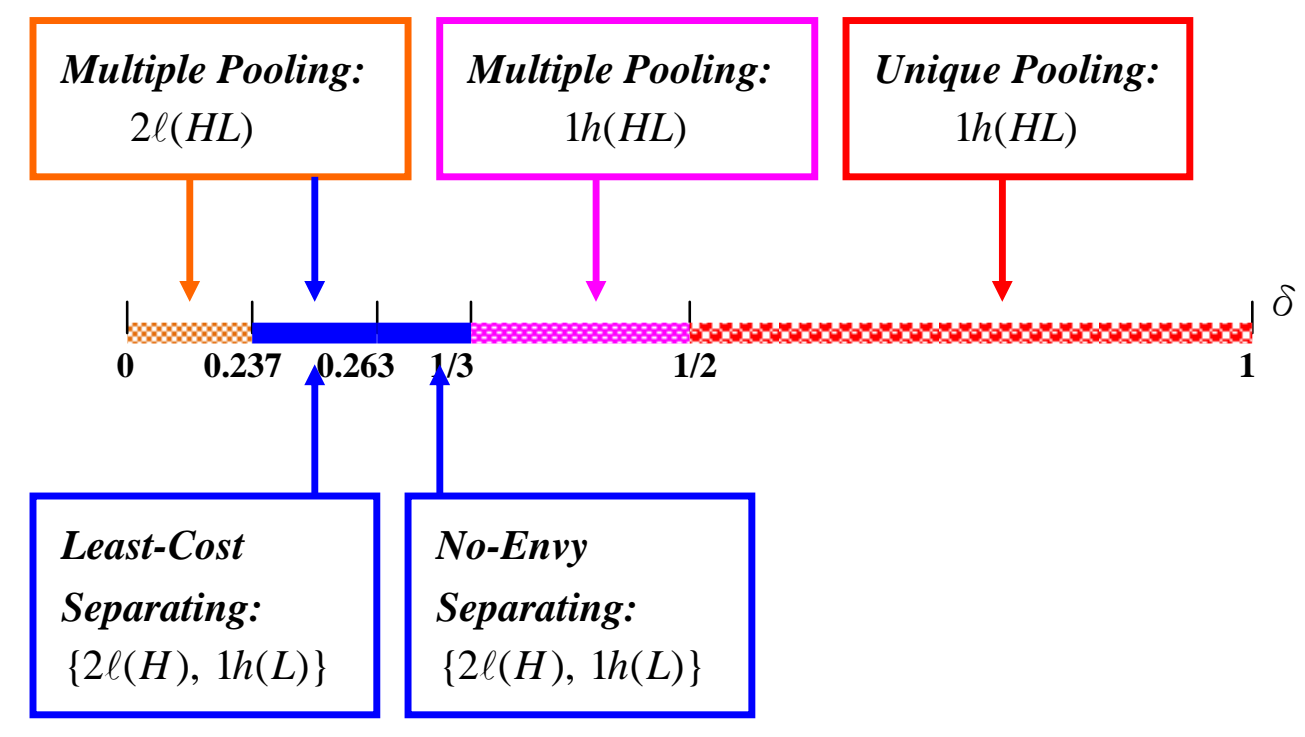

Figure 1: The Equilibrium Proposals in the Three-Session Game

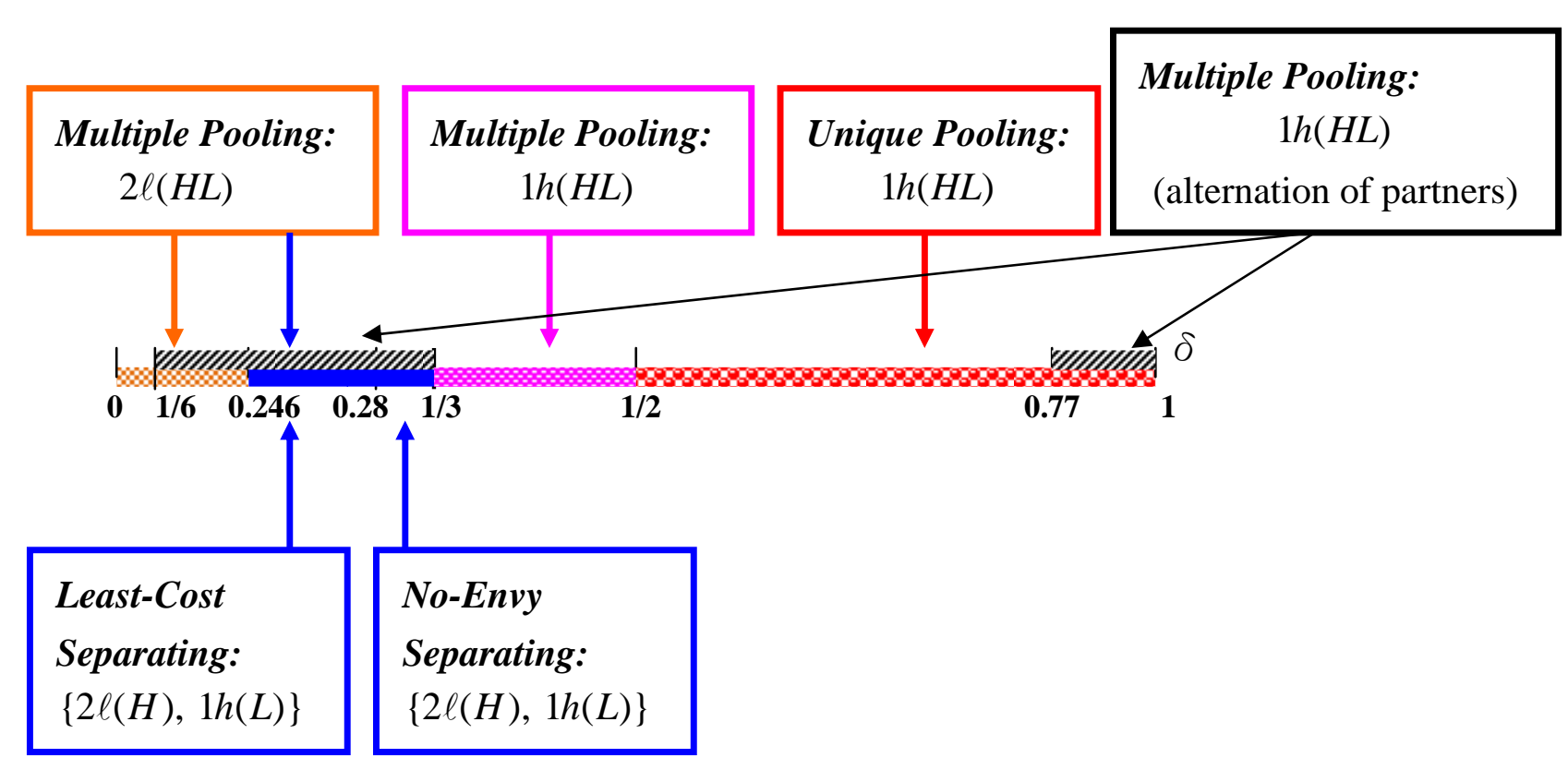

Figure 2: The Equilibrium Proposals in the Four-Session Game 